\title{
Aspects of herpes simplex virus: a clinical review
}

\author{
Alex Azwa, Simon E Barton
}

\section{Introduction}

Genital herpes $(\mathrm{GH})$ is the most frequent cause of genital ulceration worldwide. It results in a chronic, recurrent viral genital infection. It is caused by herpes simplex virus (HSV), most commonly HSV-2, although an increasing proportion of GH is now caused by HSV-1. Asymptomatic viral shedding accounts for the majority of instances of sexual transmission of HSV-2. Antiviral chemotherapy offers a significant clinical benefit to the majority of symptomatic patients and is the mainstay of management. Counselling regarding the natural history, risk of sexual and perinatal transmission, and methods to reduce transmission are integral to clinical management. Research continues to be undertaken in developing a prophylactic HSV-2 vaccine. Recent attention has focused on the role of HSV-2 as a co-factor for HIV infection and hence HSV-2 treatment may have a role as an HIV prevention strategy.

This paper reviews the epidemiology, natural history and diagnosis with emphasis on the management of $\mathrm{GH}$ in women and in pregnancy based on the latest guidelines from the British Association for Sexual Health and HIV (BASHH) and the Centers for Disease Control and Prevention (CDC) and recent published literature. It also discusses HSV-2 vaccination and the synergistic interaction between HIV and HSV, which may be driving the HIV epidemic in the developing world.

\section{Search methods}

This review is based on PubMed and MEDLINE searches for GH over the past 17 years (1990 to February 2007). Search terms used were "genital herpes, HSV, HSV epidemiology, HSV vaccine" with the limits metaanalyses, randomised controlled trial (RCT) and review. Other sources of information included the Health Protection Agency (HPA) and World Health Organization (WHO) websites and revised guidelines and recommendations of BASHH (2007), CDC (2006) and the International Herpes Management Forum. Additional data were obtained from recent international meetings and personal experience of treating patients with $\mathrm{GH}$.

\section{Epidemiology}

HSV is a double-stranded DNA virus that forms part of the alpha herpesviridae subfamily of viruses. HSV exists as two types, type 1 and 2 , which are distinguished by antigenic differences in their envelope proteins. HSV infection results in lifelong infection, which can be asymptomatic or present with recurrent lesions. A significant proportion of patients with $\mathrm{GH}$ fail to recognise their symptoms as clinical signs as these can be very subtle.

\footnotetext{
J Fam Plann Reprod Health Care 2009; 35(4): 237-242 (Accepted 28 August 2009)

Directorate of GUM and HIV Medicine, Chelsea and Westminster Hospital Foundation NHS Trust, London, UK Alex Azwa, MBChB, MRCP, Specialist Registrar

Simon E Barton, FRCOG, FRCP, Consultant
}

Correspondence to: Dr Alex Azwa, Department of GU and HIV Medicine, Chelsea and Westminster Hospital, St Stephen's Centre, 369 Fulham Road, London SW10 9NH, UK.

E-mail: alex.azwa@chelwest.nhs.uk
Recurrent lesions occur when latent virus reactivates. Generally HSV-1 has been associated with oro-labial disease with most infections occurring during childhood. HSV-2 is almost entirely associated with genital disease. However recent studies have shown that an increasing proportion of $\mathrm{GH}$ is caused by HSV-1, particularly in Europe. ${ }^{1-3}$ This may be a result of increased oral genital contact combined with lower rates of childhood oral HSV-1 and hence lower immunity to genital HSV-1 infection. Genital HSV-1 infection is associated with less severe disease and fewer symptomatic recurrences and subclinical shedding than genital HSV-2 infection. HSV-2 seroprevalence varies notably across geographical regions. Seroprevalence studies in the USA show that the rate of infection with HSV-2 has risen to $22 \%$, whereas in Europe rates between $4 \%$ and $24 \%$ have been reported; seropositivity appears to be higher in northern than in southern Europe. A UK study found that $23 \%$ of adults attending sexual health clinics, and $8 \%$ of blood donors in London, had antibodies to HSV-2. ${ }^{4} \mathrm{HSV}-2$ infection is more common in women, possibly because the rate of male-to-female transmission is at least twice that of female-to-male transmission. The prevalences of HSV genital infection increase with age and numbers of sexual partners, with higher rates in specific ethnic (eg, Black Caribbean) and low socioeconomic groups. The strongest predictor for genital HSV infection is a person's number of lifetime partners. ${ }^{5}$ In 2006, 21698 new cases were diagnosed in the UK. For females and males, highest rates were seen in the 20-24-year-olds; 208 per 100000 population and 102 per 100000 , respectively. ${ }^{6}$

\section{Natural history}

After the initial infection (primary infection) by direct contact with the mucocutaneous surfaces of an infected person, the herpes virus establishes latency in the sacral dorsal root ganglion. With reactivation, the virus travels from the dorsal root ganglion back down the nerve root resulting in either a mucocutaneous outbreak or asymptomatic shedding. It is estimated that up to $70 \%$ of all genital HSV-2 is transmitted during asymptomatic shedding from an index partner with HSV-2. The initial infection may or may not cause symptoms and is followed by seroconversion with type-specific antibodies 4-6 weeks after infection.

\section{Clinical manifestations Initial infection}

There are two types of first-episode infections. Nonprimary infections are those that occur in a patient already infected with HSV, whilst true primary infection is HSV acquisition for the first time in an HSV seronegative patient. Non-primary infections are associated with fewer systemic symptoms, a shorter duration of disease, a shorter duration of viral shedding and fewer lesions than primary infections.

After an incubation period of 1-26 days, classical primary $\mathrm{GH}$ begins with prodromal symptoms characterised by localised pain or tingling lasting up to 24 hours. Constitutional symptoms such as fever, headache, malaise and inguinal lymphadenopathy are present in twothirds of women. Papules followed by vesicles and pustules and later erosions appear over hours to days. These lesions 
usually crust and then re-epithelialise and heal without scarring. Cervical lesions are common and are almost always associated with first-episode disease. Dysuria and urinary retention may occur with urethral lesions although urinary retention is primarily associated with sacral radiculopathy.

\section{Recurrent infection}

Recurrences are generally milder than the initial episode with fewer lesions and a shorter duration of viral shedding. The median recurrence rate for HSV-2 is four recurrences per year. Approximately $90 \%$ of infected individuals have at least one recurrence during the first year, $38 \%$ have six or more recurrences and $20 \%$ have more than 10 recurrences. ${ }^{7,8}$ Recurrences are less frequent in HSV-1 and tend to decrease over time. Patients who experience severe primary symptoms have more frequent recurrences. Recurrences tend to be spontaneous but patients often report certain triggers including emotional or physical stress, menses, immunosuppression, concurrent infection and sexual intercourse.

Asymptomatic viral shedding, whereby the virus is shed intermittently on the mucosal surfaces of the genitals in the absence of clinical signs or symptoms, accounts for the majority of transmission of HSV. This means that the majority of $\mathrm{GH}$ infections are transmitted by patients unaware that they have been infected or who are asymptomatic when transmission occurs. This has implications when counselling patients regarding transmission of HSV to current or future sexual partners. The frequency of viral shedding is highest in the first year of acquisition and is less frequent with HSV-1 infection.

\section{Diagnosis \\ Viral culture}

Viral culture is the most frequently used routine diagnostic method in the UK. It allows typing of HSV isolates. It is highly specific (virtually 100\%). Sensitivity depends on the stage of the lesion and levels of viral shedding. The sensitivity of viral culture is greatest from samples obtained from patients with vesicles followed by samples from ulcers and less so in samples from crusted lesions. First-episode ulcers shed more virus than recurrent ulcers. Viral culture is, however, slow and labour intensive.

\section{Polymerase chain reaction}

Polymerase chain reaction (PCR) has greater sensitivity than viral culture. It increases HSV detection rates by $11-71 \%$ compared with viral culture. It is currently most often used for testing of cerebrospinal fluid for HSV encephalitis in patients presenting with neurological symptoms. Real-time PCR is recommended by the BASHH guidelines as the preferred diagnostic method for GH. ${ }^{9}$

Other viral antigen detection methods such as direct immunofluorescent assays are associated with a lower sensitivity and specificity than virus culture.

\section{Testing for HSV antibodies}

After infection with HSV, antibodies develop within the first few weeks. Seroconversion can take up to 12 weeks and the antibodies persist for life. Most commercial tests are not type-specific. For HSV serological assays to be useful they need to be type-specific and detect antibodies against glycoproteins $\mathrm{G}$ (gG1 and $\mathrm{gG}$ ). A positive HSV-2 serology makes the diagnosis likely especially if the patient has had lesions. A positive HSV-1 serology can make the case difficult to interpret, as it is more likely to be associated with oro-labial infection.
Western blot is the diagnostic gold standard but it is labour intensive, expensive and not widely available. The commercial glycoprotein-G-based type-specific assays that have been approved by the US Food and Drug Administration (FDA) have sensitivities varying from $80 \%$ to $98 \%$, with specificities of more than $96 \%$.

It is important to remember that the predictive value of the test is influenced by the HSV seroprevalence rates within the group being tested. The positive predictive value may be very low if the prevalence is low, even with a high sensitivity and specificity resulting in a large number of false-positives. Local epidemiological data and patient demographic characteristics should be taken into account before offering and interpreting the test.

Screening of asymptomatic patients is currently not recommended. However, the only way of identifying asymptomatic patients who have been exposed to HSV-2 infection is by serological tests. In favour of screening would be to identify HSV seropositive patients, inform them of their HSV serostatus and introduce interventions to reduce transmission to uninfected partners. These interventions include modification of sexual practices, consistent condom use and use of suppressive antiviral therapy. Confirmation of seronegative status may afford protection from vaccines in the near future. The potential harms of screening include false-positive test results and there may be huge psychological and psychosexual implications in a patient who has never experienced any symptoms in discovering they have a disease that is sexually transmissible, lifelong and one for which there is no cure. It is known that antiviral therapy improves health outcomes in symptomatic persons (eg, those with multiple recurrences), however there is no evidence that the use of antiviral therapy improves health outcomes in those with asymptomatic infection.

Circumstances for which serological screening may be useful include patients with a history of recurrent culture or PCR-negative genital ulceration or sterile cystitis or cervicitis, as well as the asymptomatic partners of patients with $\mathrm{GH}$. The latter includes asymptomatic pregnant women with male partners known to have GH. Typespecific serology can be used to identify discordant women and to introduce measures to reduce HSV acquisition. Routine screening of all asymptomatic pregnant women has been advocated especially in the USA, but appraisal of the evidence has shown that it is not cost effective except where there is a history of $\mathrm{GH}$ in the partner or demonstrating seroconversion in pregnancy. ${ }^{10}$

\section{Treatment}

\section{General treatment and advice}

All women should be told to clean affected areas with normal saline and be encouraged to pass urine in a bath if severe dysuria is present. Systemic analgesia in the form of non-steroidal anti-inflammatory drugs or paracetamol should be offered. Although local analgesia with lignocaine gel may in theory cause sensitisation, this rarely happens in practice and is often helpful particularly before micturition. It is important to look for and treat any secondary bacterial infection. All women in serodiscordant relationships should be advised to use condoms with their partners to reduce the risk of transmission and to avoid sex from the very first warning signs of an outbreak until the lesions have fully healed.

\section{Antiviral agents}

There are currently three oral antiviral drugs licensed for the treatment of GH. Aciclovir, a thymidine nucleoside analogue, was the first drug introduced to treat HSV 
infection. It has poor bioavailability and a short half-life and as a result requires frequent dosing. It is available in generic formulations. Valaciclovir is a prodrug of aciclovir and famciclovir is a prodrug of the guanosine nucleoside analogue, penciclovir. The phosphorylated active form of each drug is a competitive inhibitor of viral DNA polymerase, resulting in inhibition of viral DNA synthesis. All have similar side effects, which include nausea, vomiting, headache and diarrhoea, although these adverse effects are extremely rare.

\section{First-episode GH}

The aim of treatment is to improve symptoms and speed recovery. All the antiviral agents have been shown to reduce the duration and severity of symptoms, and reduce healing times and duration of viral shedding in primary GH. For treatment to be effective it needs to be initiated as early as possible once a clinical diagnosis has been made and before laboratory confirmation. Treatment of primary infection has not been shown to alter the natural history of the disease and has no effect on the rates of recurrences of GH. Topical agents are less effective than oral agents and combined oral and topical treatment is of no benefit.

The recommended regimens are (all for 5 days): ${ }^{9}$

1 Aciclovir $200 \mathrm{mg}$ five times daily

2 Aciclovir $400 \mathrm{mg}$ three times daily

3 Valaciclovir $500 \mathrm{mg}$ twice daily

4 Famciclovir $250 \mathrm{mg}$ three times daily.

The CDC guidelines recommend up to 10 days of treatment. ${ }^{11}$ They also recommend valaciclovir at a higher dose of $1 \mathrm{~g}$ twice daily. ${ }^{12}$ There is no evidence of benefit for courses longer than 5 days, although longer courses are justified if new lesions develop after this time.

All three drugs are similar in efficacy and toxicity hence choice would depend on cost and convenience of dosing schedule.

\section{Recurrent GH}

\section{Episodic antiviral treatment of recurrences}

Episodic treatment involves taking antiviral agents for a few days when a recurrence occurs in order to shorten the duration and severity of an episode. It is suitable for those individuals with mild and infrequent recurrences and for treating recurrences that are preceded by a prodrome. Episodic treatment treats individual recurrences, unlike suppressive treatment that aims to reduce the frequency of recurrences. To be most effective it needs to be taken during the prodromal phase or within the first 24 hours of the onset of a lesion when viral replication is at its greatest. All patients should be given a supply of antiviral agents to self-initiate as soon as a recurrence occurs. Early treatment provides a small but statistically significant benefit.

The recommended regimens are: ${ }^{9}$

1 Aciclovir $200 \mathrm{mg}$ five times daily

2 Aciclovir $400 \mathrm{mg}$ three times daily for 3-5 days

3 Valaciclovir $500 \mathrm{mg}$ twice daily

4 Famciclovir $125 \mathrm{mg}$ twice daily.

An effective course of antiviral therapy that is shorter than the current standard of 5 days is likely to improve patient compliance and overall satisfaction. A 3-day course of oral valaciclovir at a dose of $500 \mathrm{mg}$ twice daily has been shown to be as effective as a 5-day course in reducing the healing time. ${ }^{13} \mathrm{~A} 2$-day course of high-dose aciclovir at a dose of $800 \mathrm{mg}$ three times a day also has been shown to significantly reduce the duration of lesions and viral shedding when compared with placebo. ${ }^{14}$ Both the above short-course therapies have now been incorporated into the revised 2007 BASHH guidelines and the 2006 CDC guidelines for the episodic treatment of recurrent $\mathrm{GH}$.
Further studies have recently looked into reducing the duration of patient-initiated antiviral therapy even further without losing their clinical benefit leading to ultra-short courses.

Aoki et al. evaluated the effectiveness of patientinitiated single-day oral famciclovir at a dose of $1000 \mathrm{mg}$ twice daily versus placebo for the treatment of recurrent $\mathrm{GH}$ and found it to be an effective alternative to the current recommended regimens. ${ }^{15}$

Famciclovir taken within 6 hours of onset of symptoms reduced lesion healing time and duration of pain by approximately 2 days and prevented progression to a full outbreak in about one in four patients. This short-course regimen has also been now included in the current guidelines mentioned above. Bavaro et al. demonstrated that a 1-day course of oral valaciclovir at a dose of $2000 \mathrm{mg}$ twice daily in patients with recurrent $\mathrm{GH}$ was safe, well tolerated and led to a reduction in the duration of lesions and viral shedding compared to natural history studies of genital HSV-2. (NB. No placebo group was used in this study.) Only $5 \%$ of patients experienced a second lesional recurrence in the 14 days after treatment initiation. ${ }^{16}$

\section{Suppressive treatment}

This involves taking antiviral treatment continuously for a defined period of time (6 months or more) in order to prevent recurrences. It is usually recommended in patients with more than six recurrences per year or those with psychological sequelae secondary to their infection. Suppressive treatment reduces the number and frequency of symptomatic recurrences, reduces asymptomatic viral shedding and allows patients to cope better with psychological sequelae. All three drugs are likely to be equally effective and there are patient safety data available for aciclovir extending to more than 18 years. Development of resistance is extremely rare in immunocompetent patients.

Suppressive treatment has also been shown to reduce the risk of HSV transmission to uninfected partners. A landmark study by Corey et al. showed that suppressive treatment with valaciclovir $500 \mathrm{mg}$ once daily for 8 months reduced the rate of symptomatic $\mathrm{HSV}$ infection by $75 \%$ and reduced the risk of HSV-2 infection by $48 \%$ in the susceptible partner. ${ }^{17}$. Following this study, the FDA in the USA recommended that physicians offer valaciclovir therapy to immunocompetent individuals concerned about transmitting $\mathrm{GH}$ to a heterosexual partner in conjunction with condom use and safer sex behaviour.

The recommended regimens are: ${ }^{9}$

1 Aciclovir $400 \mathrm{mg}$ twice daily

2 Aciclovir $200 \mathrm{mg}$ four times daily

3 Famciclovir $250 \mathrm{mg}$ twice daily

4 Valaciclovir $500 \mathrm{mg}$ once daily.

The CDC guidelines are similar but also include valaciclovir $1 \mathrm{~g}$ once daily as an alternative option and should be considered in patients experiencing more than nine recurrences in a year. ${ }^{18}$

\section{Psychosocial implications and counselling}

For many patients, a diagnosis of $\mathrm{GH}$ infection and implications of a lifelong viral illness with recurrences can provoke severe emotions of anxiety, guilt and isolation. Counselling of infected patients and their partners is critical in the management. The goals of counselling are to help patients cope with the infection and to prevent sexual and perinatal transmission. Points to consider include: $11,19,20$

1 Patients should be informed about the natural history of the disease, potential for recurrent attacks and asymptomatic viral shedding. 
2 Antiviral therapy is available and effective but does not cure infection.

3 Patients should inform current and future sexual partners.

4 Abstain from sex when lesions or prodrome symptoms are present.

5 Sexual transmission can occur during asymptomatic periods.

6 Consistent use of latex condoms to reduce risk of transmission.

7 Sexual partners may be infected even without symptoms and serological testing can determine whether they are at risk.

8 Asymptomatic patients diagnosed by serological testing should receive the same counselling messages as those with symptomatic infections.

9 The risk of transmission can be further decreased by the daily use of valaciclovir by the infected person.

\section{Management of GH during pregnancy 20,21}

Acquisition of GH during pregnancy often causes severe anxiety to both patient and practitioner. The most devastating complication of infection is neonatal herpes, which has a high morbidity and mortality. It is rare outside North America. In the UK it has an incidence of 1.65 per 100000 live births annually compared with the much higher incidence of 20-50 per 100000 live births in the USA.

The two most important factors influencing transmission to the neonate include the timing of infection and the type of infection. A study by Brown et al. demonstrated that the greatest risk of transmission is amongst those women who acquire primary HSV infection in the third trimester and who shed the virus at term. The estimated risk of neonatal herpes with first-episode genital lesions at delivery is $31-40 \%$ compared to the smaller risk with recurrent disease of $3 \% .22$ The reasons why primary infection poses the greatest risk are two-fold. Primary infection is associated with higher viral loads and higher rates of subclinical shedding. In females who have acquired new infection in the third trimester, the neonate is not protected after intrapartum exposure by passively acquired protective maternal antibodies, which can take up to 12 weeks to develop. Other factors that facilitate neonatal transmission include use of invasive obstetric procedures such as use of fetal scalp electrodes and forceps, which may damage neonatal skin in labour and should be avoided. Duration of rupture of membranes, whereby the membranes have been ruptured by more than 4 hours, is also associated with increased risk of transmission.

\section{Prevention of neonatal HSV transmission Use of antivirals in pregnancy}

None of the three aforementioned antivirals are licensed for use in pregnancy. They are generally considered to be well tolerated and reasonably safe in pregnancy. The aciclovir pregnancy registry that collected data on prenatal exposure to aciclovir over a period of 14 years did not demonstrate any increase in the number of birth defects. ${ }^{23}$ The valaciclovir pregnancy registry that ran for 4 years and is now closed likewise did not demonstrate any increase in birth defects.

\section{First-episode GH}

\section{First- and second-trimester acquisition}

Caesarean section is not indicated in women who acquire infection in the first or second trimester. Here treatment should be with standard doses of aciclovir and vaginal delivery anticipated.

\section{Third-trimester acquisition}

Use of Caesarean section for the prevention of GH has not been evaluated in RCTs. Caesarean section should be offered to all women presenting with first-episode genital lesions at the time of delivery and in the last 6 weeks of pregnancy as the risk of viral shedding is high. Use of typespecific serology and testing of paired sera (if a booking specimen is available) can be used to identify those women with true primary infections (which carry the greater risk of transmission) and play an important role in deciding which women should proceed to having a Caesarean section. These women should also be treated with suppressive therapy with daily aciclovir in the last 4 weeks of pregnancy If vaginal delivery is unavoidable, aciclovir should be used in both mother and the baby, and use of scalp electrodes and other invasive techniques should be avoided.

\section{Recurrent GH}

Women presenting with recurrent infection should anticipate vaginal delivery unless there are lesions present at the onset of labour. Sequential viral cultures taken during late gestation do not predict viral shedding at term and are not indicated. Daily suppressive therapy with antiviral agents during the last 4 weeks of pregnancy should be offered. A recent Cochrane Database systematic review of seven RCTs assessing the effectiveness of third-trimester antiviral prophylaxis for recurrent GH with either acyclovir or valaciclovir concluded that antepartum antiviral prophylaxis reduced recurrences and viral shedding at delivery and reduced the need for Caesarean section deliveries. ${ }^{24}$ For women presenting with recurrent lesions at term, the risk of neonatal infection is small $(\sim 3 \%)$ and therefore the risks of the baby developing neonatal herpes needs to be balanced with the operative risks to the mother. Within the UK, most women with lesions at the time of delivery will be delivered by Caesarean section for medicolegal reasons.

\section{Prevention of infection acquisition in serodiscordant couples}

It is important to identify women with male partners with $\mathrm{GH}$ at their first antenatal visit. Type-specific HSV serology can be used to identify susceptible women who are at risk of acquiring infection in the later half of pregnancy where the risk of neonatal herpes is greatest. These women should then be counselled on ways they can reduce their risk of HSV acquisition. These include abstinence during their partner's recurrences and consistent use of condoms throughout pregnancy and especially during the third trimester. Pregnant women who are not infected with HSV-1 should also be advised about the risk of acquiring $\mathrm{GH}$ from oral sex with a partner with oral HSV. Suppressive therapy should be considered for the infected partner as a means of reducing HSV transmission and acquisition during pregnancy.

\section{Interaction between HIV and HSV-2}

There is increasing evidence that genital HSV-2 is a major driving force behind the HIV epidemic in sub-Saharan Africa. A meta-analysis by Freeman et al. concluded that HSV-2 infection is associated with a three-fold increase in the risk of HIV acquisition. ${ }^{25} \mathrm{HSV}$ is now widely known to facilitate HIV transmission. A prospective study among heterosexual HIV-1 discordant couples in Rakai, Uganda found that genital ulcer disease in the HIV-1 infected partner, primarily resulting from $\mathrm{HSV}-2$, was associated with a four-fold increase in the likelihood of HIV-1 transmission. ${ }^{26}$ HIV co-infection changes the natural 
history of HSV-2, resulting in more frequent and severe clinical and subclinical recurrences.

A number of observational studies have found that HSV-2 reactivation increases the levels of HIV-1 in the plasma and genital secretions in those patients co-infected with HIV-1.27,28 A recent study demonstrated that suppressive treatment with valaciclovir $500 \mathrm{mg}$ twice daily significantly reduced genital HIV shedding and plasma HIV viral loads in HSV-2/HIV-1 co-infected women. 29 However, the more recent trials to date have yielded disappointing results, ${ }^{30,31}$ although there remains hope that HSV-2 suppressive treatment can reduce HIV-1 infectiousness and delay disease progression among the HIV-1-infected individuals whose disease has not progressed to the stage at which initiation of highly active antiretroviral treatment is routinely recommended.

\section{HSV-2 vaccines}

The development of an effective prophylactic vaccine against HSV-2 has been hampered by various obstacles. ${ }^{32}$ These include complexity of the virus life cycle and establishment of latency and the various strategies that HSV possesses for evading the immune response. It has also been challenging to discover the most immunodominant protein target in a large virus with more than 80 proteins, and continued work is being undertaken to try and identify how best to stimulate the most important immune mechanisms.

Although animal studies on vaccination strategies to prevent GH may be promising, clinical trials in humans have failed to prove efficacy. The only RCTs of a vaccine in humans showing partial efficacy against genital HSV-2 to date are those of the recombinant glycoprotein vaccine, gD2-ASO4 vaccine (Simplirix ${ }^{\circledR}$ ), a gD2 glycoprotein formulated in a mixture of alum and monophosphoryl lipid A adjuvant developed by GlaxoSmithKline. 33

Subanalyses of the two large, double-blind, placebocontrolled Phase III trials of 2714 volunteers with a partner with GH disease showed that the vaccine was $73-74 \%$ effective in preventing $\mathrm{GH}$ disease but only in women who were both seronegative for both HSV-1 and HSV-2 at baseline. ${ }^{34}$ The vaccine was, however, not found to be effective in women who were previously seropositive for HSV-1 and in men regardless of their HSV serological status. This suggests that prior HSV-1 infection protects against HSV-2 disease but the vaccine did not enhance this cross-reactive immune protection. The gender difference in efficacy is interesting, and plausible explanations for this may be due to gender-specific immunological responses or possibly anatomical differences between the male and female genital mucosa.

Further Phase III trials to explore the efficacy and mechanism of the Simplirix vaccine in preventing $\mathrm{GH}$ are in progress in collaboration with the US National Institutes of Health involving over $7000 \mathrm{HSV}-1$ and HSV-2 seronegative women.

Another vaccine containing glycoprotein gB2 and gD2 formulated in the MF59 adjuvant and developed by Chiron induced high levels of neutralising antibodies at least equivalent to natural infection but failed to show efficacy in preventing $\mathrm{HSV}$ acquisition (the overall efficacy rate was $9 \%$ and higher in women, ie, 26\%). ${ }^{35}$

\section{Future perspectives of vaccines}

Further clinical research needs to be undertaken into understanding the mechanisms of the HSV immune responses, the gender-specific differences induced by the HSV-2 vaccine as shown by the earlier clinical trials, and the interaction between HSV-1 and HSV-2 to inform future vaccine development. In tandem with this, further work needs to be done to determine how best to introduce the vaccine into existing health care systems.

Several questions remain unanswered and these will largely be determined by cost-benefit analyses. Would mass vaccination or a more targeted approach be more practical? When should vaccination be started and how frequently should the vaccine be given? Targeted strategies may include vaccinating women planning a pregnancy, adolescents before the onset of sexual activity, or partners of HSV carriers. Should vaccination be led by user demand and should it be preceded by blood testing to check HSV-1 serostatus?

It is important to consider public perceptions and political barriers to immunisation against GH. Parents are the main decision makers as regards their children's health care, and the prospect of providing a vaccine to adolescent girls with the aim of preventing a sexually transmitted infection may provoke anxieties that it may encourage unsafe sex.

The interaction between HSV-1 and HSV-2 is important. Lack of efficacy of HSV-2 vaccines in HSV-1infected individuals would render the vaccine of little use in countries where the HSV-1 seroprevalence is high.

Lastly, it is widely known that HSV facilitates HIV transmission. The development of a vaccine against $\mathrm{GH}$ that might prevent HIV transmission on an individual or wider level could potentially make the vaccine more attractive.

\section{Statements on funding and competing interests}

Funding None identified.

Competing interests None identified.

\section{References}

1 Lafferty WE, Downey L, Celum C, Wald A. Herpes simplex virus type 1 as a cause of genital herpes: impact on surveillance and prevention. J Infect Dis 2000; 181: 1454-1457.

2 Strutt M, Bailey J, Tenant-Flowers M, Graham D, Zuckerman $M$. Ethnic variation in type of genital herpes simplex virus infection in a South London genitourinary medicine clinic. J Med Virol 2003; 69: 108-110.

3 Scoular A, Norrie J, Gillespie G, Mir N, Carman WF. Longitudinal study of genital infection by herpes simplex virus type 1 in Western Scotland over 15 years. BMJ 2002; 324: 1366-1367.

4 Cowan FM, Johnson AM, Ashley R, Corey L, Mindel A. Antibody to herpes simplex virus type 2 as a serological marker of sexual lifestyle in populations. BMJ 1994; 309: 1325-1329.

5 Corey L, Handsfield HH. Genital herpes and public health: addressing a global problem. JAMA 2000; 283: 791-794.

6 Health Protection Agency. Genital herpes statistics. http://www.hpa.org.uk/hpr/archives/2009/news2909.htm [Accessed 21 August 2009].

7 Benedetti J, Corey L, Ashley R. Recurrence rates in genital herpes after symptomatic first episode infection. Ann Intern Med 1994; 121: 847-854.

8 Beauman JG. Genital herpes: a review. Am Fam Physician 2005; 72: 1527-1534.

9 British Association for Sexual Health and HIV. 2007 National Guideline for the Management of Genital Herpes. http://www.bashh.org/documents/115/115.pdf [Accessed 21 August 2009].

10 Tita AT, Grobman WA, Rouse DJ. Antenatal herpes serologic screening: an appraisal of the evidence. Obstet Gynecol 2006; 108: $1247-1253$.

11 Centers for Disease Control and Prevention. Sexually Transmitted Diseases Treatment Guidelines 2006: Genital HSV Infections. http://www.cdc.gov/std/treatment/2006/genitalulcers.htm\#genulc3 [Accessed 21 August 2009].

12 Fife KH, Barbarash RA, Rudolph T, Degregorio B, Roth R. Valaciclovir versus acyclovir in the treatment of first -episode genital herpes infection. Results of an international, multicenter, double-blind randomized clinical trial. The Valaciclovir International Herpes Simplex Virus Study Group. Sex Transm Dis 1997; 24: 481-486.

13 Leone PA, Trottier S, Miller JM. Valacyclovir for episodic treatment of genital herpes: a shorter 3-day treatment course 
compared with 5-day treatment. Clin Infect Dis 2002; 34: 958-962.

14 Wald A, Carrell D, Remington M, Kexel E, Zeh J, Corey L. Twoday regimen of acyclovir for treatment of recurrent genital herpes simplex virus type 2 infection. Clin Infect Dis 2002; 34: 944-948.

15 Aoki FY, Tyring S, Diaz-Mitoma F, Gross G, Gao J, Hamed K Single-day patient-initiated famciclovir therapy for recurrent genital herpes: a randomized, double-blind, placebo-controlled trial. Clin Infect Dis 2006; 42: 8-13.

16 Bavaro JB, Drolette L, Koelle DM, Almekinder J, Warren T, Tyring $S$, et al. One-day regimen of valaciclovir for treatment of recurrent genital herpes simplex virus 2 infection. Sex Transm Dis 2008; 35: 383-386.

17 Corey L, Wald A, Patel R, Sacks SL, Tyring SK, Warren T, et al; Valacyclovir HSV Transmission Study Group. Once-daily valacyclovir to reduce the risk of transmission of genital herpes. N Engl J Med 2004; 350: 11-20.

18 Reitano M, Tyring S, Lang W, Thoming C, Worm AM, Borelli S, et al. Valaciclovir for the suppression of recurrent genital herpes simplex virus infection: a large-scale dose range-finding study. International Valaciclovir HSV Study Group. J Infect Dis 1998; 178: 603-610.

19 International Herpes Management Forum. http://www.ihmf.org/ [Accessed 21 August 2009].

20 Sen P, Barton SE. Genital herpes and its management: clinical review. BMJ 2007; 334: 1048-1052.

21 Royal College of Obstetricians and Gynaecologists. Management of Genital Herpes in Pregnancy (Green-top Guideline No. 30). September 2007. http://www.rcog.org.uk/ womens-health/clinical-guidance/management-genital-herpespregnancy-green-top-30 [Accessed 21 August 2009]

22 Brown ZA, Benedetti J, Ashley R, Burchett S, Selke S, Berry S, et al. Neonatal herpes simplex virus infection in relation to asymptomatic maternal infection at the time of labour. $N$ Engl $J$ Med 1991; 324: 1247-1252.

23 Reiff-Eldridge R, Heffner CR, Ephross SA, Tennis PS, White $A D$, Andrews EB. Monitoring pregnancy outcomes after prenatal drug exposure through prospective pregnancy registries: a pharmaceutical company commitment. $A m ~ J$ Obstet Gynecol 2000; 182: 159-163.

24 Hollier LM, Wendel GD. Third trimester antiviral prophylaxis for preventing maternal genital herpes simplex virus (HSV) recurrences and neonatal infection Cochrane Database Syst Rev 2008; 1: CD004946.
25 Freeman EE, Weiss HA, Glynn JR, Cross PL, Whitworth JA, Hayes RJ. Herpes simplex virus 2 infection increases HIV acquisition in men and women: systematic review and metaanalysis of longitudinal studies. AIDS 2006; 20: 73-83.

26 Gray RH, Wawer MJ, Brookmeyer R, Sewankambo NK, Serwadda D, Wabwire-Mangen $\mathrm{F}$, et al. Probability of HIV-1 transmission per coital act in monogamous, heterosexual, HIV1-discordant couples in Rakai, Uganda. Lancet 2001; 357: 1149-1153.

27 Schacker T, Zeh J, Hu H, Shaughnessy M, Corey L. Changes in plasma human immunodeficiency virus type 1 RNA associated with herpes simplex virus reactivation and suppression. J Infect Dis 2002; 186: 1718-1725.

28 Celum CL, Robinson NJ, Cohen MS. Potential effect of HIV type 1 antiretroviral and herpes simplex virus type 2 antiviral therapy on transmission and acquisition of HIV type 1 infection. $J$ Infect Dis 2005; 191(Suppl. 1): S107-S114.

29 Nagot N, Ouedraogo A, Mayaud P, Konate I, Vergne L, Weiss $\mathrm{HA}$, et al. Reduction of HIV-1 RNA levels with therapy to suppress herpes simplex virus. N Engl J Med 2007; 356: 790-799.

30 Watson-Jones D, Weiss HA, Rusizoka M, Changalucha J, Baisley K, Mugeye K, et al; HSV Trial Team; Steering and Data Monitoring Committees. Effect of herpes simplex suppression on incidence of HIV among women in Tanzania. N Engl J Med 2008; 358: 1560-1571.

31 Celum C, Wald A, Hughes J, Sanchez J, Reid S, DelanyMoretlwe S, et al; HPTN 039 Protocol Team. Effect of aciclovir on HIV-1 acquisition in herpes simplex virus 2 seropositive women and men who have sex with men: a randomised, double-blind, placebo-controlled trial. Lancet 2008; 371: 2109-2119.

32 Jones CA, Cunningham AL. Vaccination strategies to prevent genital herpes and neonatal herpes simplex virus disease. Herpes 2004: 11: 12-17.

33 Haddow LJ, Mindel A. Genital herpes vaccines-cause for cautious optimism. Sex Health 2006, 3: 1-4.

34 Stanberry LR, Spruance SL, CunninghamAL, Bernstein DI, Mindel A, Sacks S, et al. Glycoprotein-D-adjuvant vaccine to prevent genital herpes. N Engl J Med 2002; 347: 1652-1661.

35 Corey L, Langenberg AG, Ashley R, Sekulovich RE, Izu AE, Douglas JM, et al. Recombinant glycoprotein vaccine for the prevention of genital HSV-2 infection: two randomised controlled trials. Chiron HSV Vaccine Study Group. JAMA 1999; 282: 331-340.

BOOK REVIEWS

Speakeasy: Talking With Your Children About Growing Up. Family Planning Association (with a foreword by Dr Miriam Stoppard). London, UK: fpa, 2009. ISBN-13: 978-1-905506-63-7. Price: £9.99. Pages: 132 (paperback)

Question 1: Where did young people get most sex and relationships education (SRE) in the 20th century? Answer: According to a British School Inspectorate (OFSTED) survey, from parents. Question 2: Where do young people get most SRE in the 21st century? Answer: According to OFSTED, from teachers

So why the shift? All too often because parents and carers feel embarrassed, de-resourced, powerless in a society so sexualised that it sometimes feels as if children know more about sex than adults do. Which is where the fpa's 'Speakeasy' courses come in, helping such adults to give young people accurate and supportive sex education in the family setting.

But why am I wittering on about face-to-face courses in the middle of a book review? The answer is that the fpa has now turned the course material into book form, based on the experience of Speakeasy trainers and participants.

And it works. The book is outstandingly practical, clearly based on the real problems of real parents, and on real solutions that have worked. It begins by addressing why home SRE is vital, how to overcome embarrassment, how to start talking, There follows coverage of puberty, love, sexuality, safer sex, pornography, contraception, STIs, pregnancy choices and pregnancy safety.

This is all presented through simple text with numerous suggestions of 'how to'; what questions you can ask young people, what questions they may ask and how to answer, and sample problem scenarios. Throughout, the material takes into account different religious and cultural issues; different age groups of children; and what to do if you are a step-parent, grandparent or foster parent. The whole book is written simply, directly, personally and without a hint of judgement or patronising tone.

Any criticisms? Only trivial ones. The big format and large print slightly hint at 'textbook'. The reams of useful organisations are - as might be expected - UK-based.

But I'm quibbling. My end judgement is that this book should be recommended by any Journal reader to every parent or carer - and read by every Journal reader who has children. I further hold that if, next time OFSTED assess where sex education is being delivered, they discover that home SRE has risen in the league tables, then this book will be hugely responsible for that shift having occurred.

The Complete Idiot's Guide to Sexual Health and Fitness. Kate Bracey, Kathryn Arendt, David Winchester. London, UK: Alpha Books (Penguin Group), 2008, ISBN-13: 978-1-592-57766-8. Price: £11.40. Pages: 320 (paperback)

I like the Complete Idiot's Guides that I've previously seen. Their format makes for easy accessibility and their informal, slightly quirky approach makes for an enjoyable reading experience. So I was entirely prepared to like this new publication.

And in many respects I wasn't disappointed. The structure is ideal for the 'dip in' nature of most sexual health queries. The layout is clear, slightly magazine-like and therefore easy to read. The facts are generally accurate, up-to-date and highly useful. The later, more factual chapters cover the information ground comprehensively.
My objection is the fact that all too often the style descends into what I can only describe as 'hectoring', particularly in the earlier, less factual chapters. The authors seem mildly irritated with their audience - with the individual reader of the book but also, I suspect, with the sexual health consumer in general. Hence the book is full of en passant diatribes. "Stop measuring yourself against some arbitrary standard", "You only have to be wrong about [protection] once to pay the price", "One day you are going to wake up a really disappointed person with ... a new case of herpes", and so on.

This tone concerns me. Of course we want patients to understand the risks they take with regard to sexuality. But we don't want them to feel cowed which, frankly, was the way I felt after reading some parts of this book. I would much rather the authors had tempered their warnings with an attempt to get the reader 'on side' rather than stressing so often what mistakes the reader is making.

Hence, in summary, I can absolutely recommend this as a source book for more formal settings: to keep in a practice library for instant reference, or to support a confident patient who simply needs extra information. But for young people and vulnerable patients, I'd prefer to recommend something a little more sensitive and compassionate.

In short, while patients may need the facts and insights this Complete Idiot's Guide offers, they don't need to be treated like complete idiots, which is, sadly, what this book often does.

Reviewed by Susan Quilliam, BA, MNLP

Freelance Writer, Broadcaster and Agony Aunt, Cambridge, $U K$ 